\title{
Investigation of Grain Growth and Deformation in Nanocrystalline Metals Through In-situ TEM Mechanical Testing and Crystallographic Orientation Mapping
}

\author{
Christopher M. Barr ${ }^{1}$, Daniel C. Bufford ${ }^{1}$ and Khalid Hattar ${ }^{1}$ \\ 1. Sandia National Laboratories, Radiation-Solid Interactions, Albuquerque NM, USA
}

Evaluation of the long term mechanical response of nanocrystalline alloys is critical for potential use in structural engineering applications. While nanocrystalline metals have indicated increased fatigue and yield strength in comparison to coarse grain metals, there are a number of limiting factors including undesired mechanical and/or thermal induced grain growth as well as limited strain hardening capacity. To evaluate and correlate microstructural evolution during deformation in nanocrystalline metals, in-situ TEM mechanical testing has provided a number of useful insights on both grain boundary and dislocation based plasticity [1-3]. This paper demonstrates efforts to correlate localized dislocation and grain boundary evolution during in-situ deformation using both qualitative and quantitative mechanical testing approaches in a range of nanocrystalline FCC metals. Specific emphasis is placed on methodologies to correlate localized crystallographic orientations and grain growth during in-situ TEM mechanical testing for improved structure-property relationships.

Qualitative in-situ TEM mechanical testing was performed on nanocrystalline Ni and Pt. The mechanical loading was performed in a Gatan single tilt straining holder at room temperature. The samples were prepared from either magnetron sputtering or pulse laser deposition and deposited on single crystal $\mathrm{NaCl}$ substrates. Thin films were floated off of $\mathrm{NaCl}$ substrates onto custom designed loading substrate holders. The microstructural evolution was tracked during the tensile loading after propagating nanoscale cracks formed in the thin films. In particular experiments, when a sufficiently slow propagating crack formed, successive precession enhanced automated crystallographic orientation maps (ACOM-TEM) and bright field TEM imaging were used to examine the microstructural evolution in front of the crack tip evolution. A number of localized microstructural observations were documented near the crack tip during in-situ tensile loading. In Pt, twinning/de-twinning was observed during momentary crack tip arrest. A sequence of images from the potential twinning and de-twinning observation at the crack tip is shown in Figure 1. Figure $1 \mathrm{~b}$ indicates the rapid formation of a potential twin boundary at the crack tip while Figure 1d indicates after approximately 120 seconds, the twin has been removed. Shortly after the twin is removed, the crack tip is shown to propagate in an intergranular fashion. Further localized changes in the microstructural evolution in front of the crack tip in nanocrystalline Pt include grain elongation and possible grain growth coupled with significant in-grain dislocation activity as shown in Figure 2 . In parallel with the nanocrystalline $\mathrm{Pt}$, similar experiments performed with nanocrystalline Ni indicate evidence of a discontinuous grain growth process in front the crack tip evolution.

While qualitative mechanical testing can provide insight into dislocation and grain boundary mediated plasticity, quantitative mechanical testing provides improved load and displacement control. Our recent efforts have indicated the ability to measure high cycle fatigue crack growth rates coupled with evidence of grain growth in nanocrystalline $\mathrm{Cu}$ through combined cyclic loading and ACOM-TEM [3]. The ability to couple controlled mechanical testing and ACOM-TEM provides a clear direction forward for improved fundamental understanding of mechanical stability in nanocrystalline metals under various loading regimes [4]. 


\section{References:}

[1] M. Legros, D.S. Gianola, K.J. Hemker, Acta Materialia 56 (2008), p. 3380.

[2] A Kobler et al, Ultramicroscopy 128 (2013), p. 68.

[3] D.C. Bufford et al, Nano Letters 16 (2016), p. 4946.

[4] This work was fully supported by the Division of Materials Science and Engineering, Office of Basic Energy Sciences U.S Department of Energy. Sandia National Laboratories is a multi-program laboratory managed and operated by Sandia Corporation, a wholly owned subsidiary of Lockheed Martin Corporation, for the U.S. Department of Energy's National Nuclear Security Administration under contract De-AC04-94AL85000.
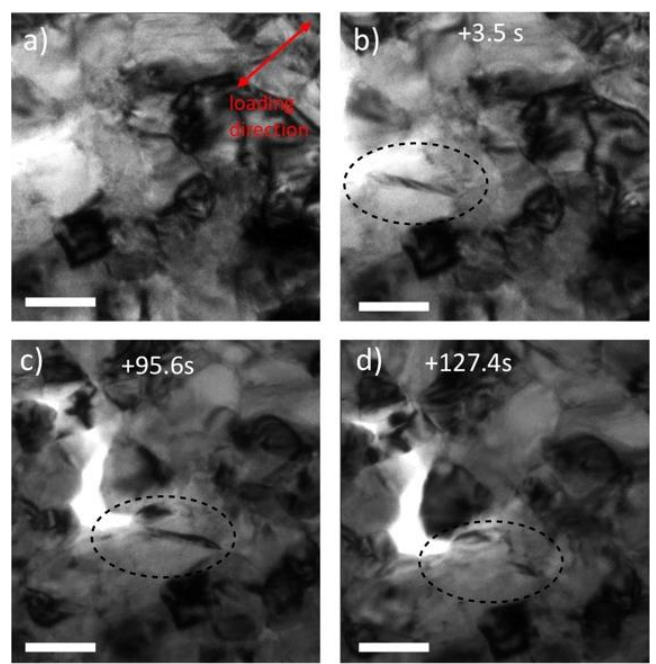

Figure 1. Observation of possible twinning/de-twinning event at dashed circle between a sequence of images over 127 seconds (a-d) at an arrested crack tip in nanocrystalline Pt under tensile load. Scale bar is $40 \mathrm{~nm}$.
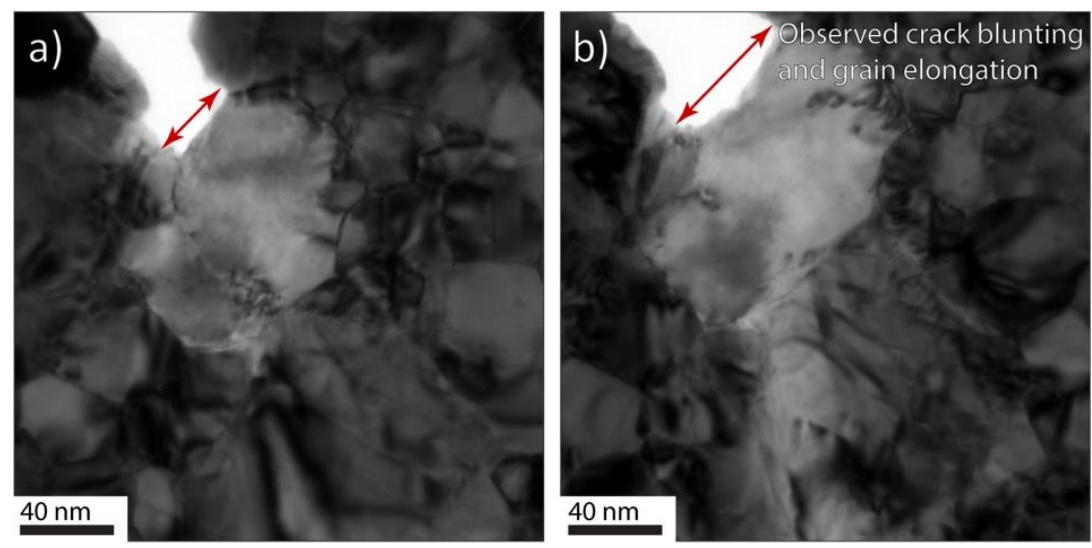

Figure 2. Observation of crack tip blunting between initial crack tip (a) and after multiple loading pulses (b). The tip blunting in nanocrystalline $\mathrm{Pt}$ is shown to be associated approximately $75 \%$ grain elongation during in-situ mechanical. 